\title{
A BICOMBING THAT IMPLIES A SUB-EXPONENTIAL ISOPERIMETRIC INEQUALITY
}

\author{
by GÜNTHER HUCK and STEPHAN ROSEBROCK
}

(Received 2nd March 1992)

\begin{abstract}
The idea of applying isoperimetric functions to group theory is due to $\mathrm{M}$. Gromov [8]. We introduce the concept of a "bicombing of narrow shape" which generalizes the usual notion of bicombing as defined for example in [5], [2], and [10]. Our bicombing is related to but different from the combings defined by $M$. Bridson [4]. If they Cayley graph of a group with respect to a given set of generators admits a bicombing of narrow shape then the group is finitely presented and satisfies a sub-exponential isoperimetric inequality, as well as a polynomial isodiametric inequality. We give an infinite class of examples which are not bicombable in the usual sense but admit bicombings of narrow shape.
\end{abstract}

1991 Mathematics subject classification: 20F05, 05C25.

\section{Definitions}

Let $\Gamma_{X}(G)$ be the Cayley graph of a group $G$ with respect to a finite set of generators, $X$, and let $\Gamma_{X}(G)$ be equipped with the word metric. Let $F$ be the free group on $X$. For $v \in F$ let $|v|$ denote the length in the free group.

A bicombing as defined in [2] and [10] is essentially a selection of a path $\sigma(g, h)$ for every pair of vertices $g, h \in \Gamma_{X}(G)$, such that the distance between any two paths which start and end a distance $\leqq 1$ apart is uniformly bounded. We replace the uniform bound for this distance by a bound that is dependent on the lengths of the paths. More precisely, we define a bicombing of narrow shape as follows:

For each $(g, h) \in G \times G$ let $\sigma(g, h):\left[0, \infty\left[\rightarrow \Gamma_{X}(G)\right.\right.$ be a path from $g$ to $h$ which is at integer times at vertices (i.e. from $t=n$ to $t=n+1$ the path either travels the distance between two adjacent vertices or pauses at a vertex). We define the length:

$$
|\sigma(g, h)|=\min \{t \mid \sigma(g, h)[t, \infty[=\text { constant }=h\} .
$$

This is the length of the path including the pauses which occur before the end of the path is reached. We will frequently represent such a path by a sequence of elements in $X \cup X^{-1} \cup\{1\}$ which, given the start vertex $g$, completely determines the path. Let $\sigma(h)=\sigma(1, h)$. We call $\sigma$ a bicombing of narrow shape if

(1) it is "recursive", i.e. if there exists an increasing polynomial $f: \mathbb{N} \rightarrow \mathbb{N}$, such that

$$
|\sigma(g)| \leqq f(d(1, g)) \forall g \in G
$$

(2) there exists an integer $M>1$ and a real number $k>2$, such that for any $g \in G$ $|\sigma(g, g)| \leqq M k / 2$ and for all $g, h \in G$ and $a, b \in X^{ \pm 1} \cup\{1\}$ 


$$
|\sigma(\sigma(g, h)(t), \sigma(g a, h b)(t))| \leqq \max ((|\sigma(g, h)|+|\sigma(g a, h b)|) / k, M / 2)
$$

holds for all integers $t \in[0, \infty[$.

where $d(1, g)$ denotes the distance in $\Gamma_{X}(G)$ from 1 to $g$. If possible we will always choose $\sigma(1)$ to be the identical path. A bicombing is called geodesic if $f$ is the identity (i.e. the combing lines are geodesics).

Let the group $G$ be finitely generated with generator set $X$. Following Gersten [7], a function $f: \mathbb{N} \rightarrow \mathbb{N}$ is called an isoperimetric function for $G$ if for any word $w$ in $X$ of length $n$ with $w=1$ in $G$, the minimum number of 2-cells in a van Kampen diagram for $w$ is at most $f(n)$.

Let $P=\langle X \mid R\rangle$ be a finite presentation of the group G. Following Gersten [7], a function $f: \mathbb{N} \rightarrow \mathbb{R}$ is called an isodiametric function for $P$, if for any word $w$ in the generators $X$ with $w=1$ in $G$ there is a van Kampen diagram for $w$, such that any vertex in the diagram has distance at most $f(|w|)$ from the basepoint.

We would like to thank Allan J. Sieradsky, Holger Meinert, Stephen J. Pride, William A. Bogley and all the members of our "Luttach workshop" for helpful discussions.

\section{An isoperimetric inequality and an isodiametric function}

Theorem 2.1. A group $G$ with finite generator set $X$ and a bicombing of narrow shape is finitely presented and has an isoperimetric function of growth $n^{0(\log n)}$.

Proof. Define a presentation $P=\langle X \mid R\rangle$, where $R$ is the set of all cyclically reduced non-trival words of length at most $M+2$ which are trivial in $G$. We prove that $P$ is a presentation for $G$ by constructing a van Kampen diagram for each word which is trivial in $G$, using only 2 -cells of $R$.

Let $w \in F$ be a reduced nontrivial word of length $n>M+2$ which is trivial in $G$. If $w=x_{1} \ldots x_{n}, x_{i} \in X^{ \pm 1}$, define $w_{i}=x_{1} \ldots x_{i}$. Now consider the "fan" of bicombing lines $\sigma\left(w_{i}\right)$ from 1 to $w_{i}$. The equality $|w|=n$ implies $d\left(1, w_{i}\right) \leqq n / 2$ and by (1) it follows that

$$
\left|\sigma\left(w_{i}\right)\right| \leqq f(n / 2) \quad \text { for } 1 \leqq i \leqq n
$$

If $\left|\sigma\left(w_{i}\right)\right|+\left|\sigma\left(w_{i+1}\right)\right| \leqq M$, then the closed path $\tau_{i}=\sigma\left(w_{i}\right) x_{i+1} \sigma\left(w_{i+1}\right)^{-1}$ in $\Gamma_{X}(G)$ is of length $\leq M+2$ and therefore represents up to cyclic reduction an element of $R$.

If $\left|\sigma\left(w_{i}\right)\right|+\left|\sigma\left(w_{i+1}\right)\right|>M$ we break up the closed path $\tau_{i}$ again, using the bicombing paths $\sigma_{i, t}=\sigma\left(\sigma\left(w_{i}\right)(t), \sigma\left(w_{i+1}\right)(t)\right)$ that connect $\sigma\left(w_{i}\right)(t)$ to $\sigma\left(w_{i+1}\right)(t)$ for all positive integers $t \leqq \max \left(\left|\sigma\left(w_{i}\right)\right|,\left|\sigma\left(w_{i+1}\right)\right|\right)$. By (2),

$$
\left|\sigma_{i, \ell}\right| \leqq \max (2 f(n / 2) / k, M / 2) .
$$

Let $\sigma\left(w_{i}\right)=a_{1} \ldots a_{p}, \sigma\left(w_{i+1}\right)=b_{1} \ldots b_{q}, a_{j}, b_{l} \in X^{ \pm 1} \cup\{1\}$. We examine the length of the closed paths $\tau_{i, t}$ that are generated by the connecting paths $\sigma_{i, t}: \tau_{i, t}=\sigma_{i, t} b_{t+1} \sigma_{i, t+1}^{-1} a_{t+1}^{-1}$ (see fig. 1). If $\left|\sigma_{i, 1}\right|+\left|\sigma_{i, \ell+1}\right| \leqq M$, then $\left|\tau_{i, 1}\right|<M+2$ and $\tau_{i, \ell}$ represents up to cyclic 


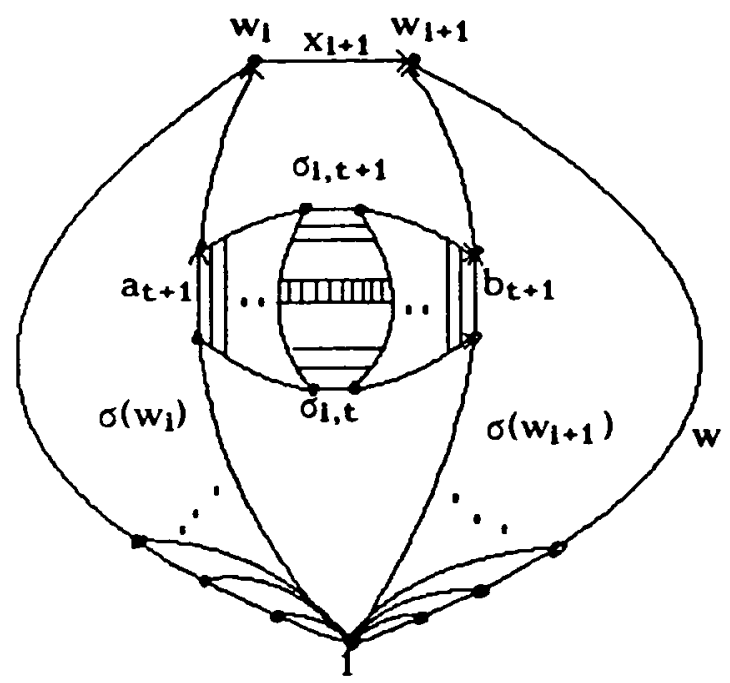

FIGURE 1. A diagram for $w$.

reduction an element in $R$. Otherwise, we break $\tau_{i, t}$ up agian using bicombing paths $\sigma_{i, t, s}=\sigma\left(\sigma_{i, t}(s), \sigma_{i, t+1}(s)\right)$ for $s \leqq \max \left(\left|\sigma_{i, t}\right|,\left|\sigma_{i, t+1}\right|\right)$.

There is one exception, namely if we are close to the boundary. This is because the path of length one between $w_{i}$ and $w_{i+1}$ is not (necessarily) a combing line. But the condition $|\sigma(g, g)| \leqq M k / 2$ implies

$$
\left|\sigma\left(\sigma\left(w_{i}, w_{i}\right)(0), \sigma\left(w_{i+1}, w_{i+1}\right)(0)\right)\right| \leqq \max \left(\frac{\left|\sigma\left(w_{i}, w_{i}\right)\right|+\left|\sigma\left(w_{i+1}, w_{i+1}\right)\right|}{k}, \frac{M}{2}\right) \leqq M,
$$

and the closed path on the boundary consisting of the combing line $\sigma\left(w_{i}, w_{i+1}\right)$ and the edge from $w_{i+1}$ to $w_{i}$ has length $\leqq M+1$ and therefore represents an element of $R$.

By (4), $\left|\sigma_{i, t, s}\right| \leqq \max \left(4 f(n / 2) / k^{2}, M / 2\right)$. If $\left|\sigma_{i, t, s}\right|+\left|\sigma_{i, t, s+1}\right| \leqq M$ then the closed path $\tau_{i, t, s}$, using $\sigma_{i, t, s}, \sigma_{i, t, s+1}^{-1}$ and the segments of length $\leqq 1$ along $\sigma_{i, t}$ and $\sigma_{i, s+1}$, is of length $\leqq M+2$ and therefore represents an element in $R$. Otherwise, we break up further in the same manner using connecting bicombing paths of length $\leqq$ $\max \left(8 f(n / 2) / k^{3}, M / 2\right)$, etc. until $2^{d} f(n / 2) / k^{d} \leqq M / 2$. In this way we find a van Kampen diagram for $w$. This proves that $G$ is finitely presented. The exponent $d$ can be estimated as the smallest integer greater than or equal to $\log _{k / 2}(2 f(n / 2) / M)$.

The isoperimetric inequality has the form:

$$
\begin{gathered}
\#(2-\text { cells }) \leqq n \cdot( \\
f(n / 2)+1) \cdot 2(f(n / 2)+2) / k \cdots 2^{d-1}(f(n / 2)+2) / k^{d-1} \\
\leqq \frac{n(f(n / 2)+2)^{d} 2^{d(d-1) / 2}}{k^{d(d-1) / 2}}=n^{0(\log n)}
\end{gathered}
$$


where $d$ is given as above.

Remark 1. Condition (1) is not necessary in order to prove that the presentation is finite.

2. The growth of the isoperimetric function is faster than polynomial but slower than exponential; therefore we call it sub-exponential.

Theorem 2.2. Each group that has a bicombing in the sense of [10] has a bicombing of narrow shape.

Proof. By using the notation of the proof above, the bicombing in the sense of Short is a narrow bicombing with $\left|\sigma_{i, t}\right| \leqq M / 2$ and $f(n)=m n$ for a given constant $m \in \mathbb{N}$ and $d=1$ in this case.

Theorem 2.3. Let $P=\langle X \mid R\rangle$ be a finite presentation for the group $G$ with a bicombing of narrow shape $\sigma$ and let $f$ be the polynomial from (l) bounding $|\sigma(g)|$.

(1) There is a polynomial isodiametric function for $P$ of the same degree as $f$.

(2) If $\sigma$ is geodesic, then the isodiametric function is linear.

Proof. Let $w \in F$ be a reduced nontrivial word of length $n$, which is trivial in $G$, and let $D$ be the van Kampen diagram for $w$ constructed in the proof of Theorem 2.1. One can reach every vertex in the diagram $D$ from the basepoint 1 by travelling part of a bicombing line $\sigma\left(w_{i}\right)$ of the first generation then travelling part of a bicombing line $\sigma_{i, t}$ of the second generation then part of a bicombing line $\sigma_{i, t, s}$ of the third generation etc. The length of a bicombing line of the lth generation is $\leqq 2^{i} f(n / 2) / k^{l}$, and the sum of the lengths of successive generations of bicombing lines therefore is $\leqq$ $f(n / 2)\left(1+2 / k+(2 / k)^{2}+\cdots\right)=f(n / 2) k /(k-2)$. Hence $(k /(k-2)) f(n / 2)$ is an isodiametric function for the presentation $P$. If $\sigma$ is geodesic, then $f$ is the identity and the above function is linear.

The next theorem follows an idea of $M$. Bridson [4]. It shows that the definition of a bicombing of narrow shape cannot be sharpened.

Theorem 2.4. Let $X$ be a finite generating set of the group $G$. Choose for every pair $g, h \in G$ a geodesic $\sigma(g, h) \in \Gamma_{X}(G)$. Then

$$
\forall x, y \in X^{ \pm 1}, \quad \forall g, h \in G,|\sigma(\sigma(g, h)(t), \sigma(g x, h y)(t))| \leqq(|\sigma(g, h)|+|\sigma(g x, h y)|) / 2+1
$$

holds for all integers $t \in[0, \infty[$.

Proof. Let $C=(|\sigma(g, h)|+|\sigma(g x, h y)|) / 2$. If $t \leqq C / 2$, then following $\sigma(g, h)$ backwards from $\sigma(g, h)(t)$ to $g$ then one edge to $g x$ and then going to $\sigma(g x, h y)(t)$ along $\sigma(g x, h y)$ 
gives a path of length at most $C+1$. For $t>C / 2$ follow $\sigma(g, h)$ from $\sigma(g, h)(t)$ to the vertex $h$, then go one edge to $h y$ and then to $\sigma(g x, h y)(t)$ backwards along $\sigma(g x, h y)$. This gives a path of length at most $C$.

\section{A class of examples}

Let $P_{q}=\left\langle x, y, z \mid\left[x, y^{q}\right]=z,[x, z]=[y, z]=1\right\rangle$ be a presentation of the group $G_{q}$ where $q \geqq 1$ and $[a, b]$ denotes the commutator of $a$ and $b . G_{1}$ is the 3-dimensional integral Heisenberg group. Let $F$ be the free group on $\{x, y, z\}$. Let $w, v \in F$. If both words are equal in $F$, we write $w \equiv v$. If they are the same in $G_{q}$, we write $w=v$.

It is easy to see, that

$$
z^{j l}=x^{j} y^{q l} x^{-j} y^{-q l}
$$

holds in $G_{q}$.

Lemma 3.1 (normal form for $G_{q}$ ). Let $w \in F$. Then, for $q>1$, there is a word

$$
\tau(w) \equiv y^{s} x^{r_{1}} y^{s_{1}} x^{r_{2}} \ldots y^{s_{m-1}} x^{r_{m}} y^{p} z^{n} \in F
$$

with $r_{i}, s_{i} \neq 0$ and

$$
\text { for } q \text { even: } s, s_{i} \in\{-q / 2+1, \ldots, q / 2\} \text {, }
$$

for $q$ odd: $s, s_{i} \in\{-(q-1) / 2, \ldots,(q-1) / 2\}$,

and, for $q=1$, there is a word

$$
\tau(w) \equiv x^{r} y^{p} z^{n} \in F
$$

such that $\tau(w)=w$ in $G_{q}$ and for all $v \in F$ with $w=v$ in $G_{q}, \tau(w) \equiv \tau(v)$.

Proof. The case $q=1$ is trivial. For $q>1$ it is easy to see that each word $w \in F$ can be transformed into $\tau(w)$ using the relations of $\boldsymbol{P}_{q}$. In order to prove uniqueness, let $\boldsymbol{w}$ and $v$ be two words in $F$ representing the same element in $G_{q}$. Let $H_{q}=G_{q} / \ll z \gg$, where $\ll z \gg$ denotes the normal closure of $z$ in $G_{q} . T_{q}=\left\langle x, y \mid x y^{q} x^{-1}=y^{q}\right\rangle$ is a presentation for $H_{q}$, which is an HNN-extension. Therefore $w$ and $v$ have the same normal form (see [9]) $\tau^{\prime}(w)=\tau^{\prime}(v)$ in $H_{\mathrm{q}}$ which is equal to the normal form in $G_{q}$, except that $n=0$. Since $z$ is central, $\tau(w)$ and $\tau(v)$ can only differ by a power of $z$. But $z$ has infinite order in $G_{q}$ which implies $\tau(v) \equiv \tau(w)$.

The normal forms (6) and (7) define a path $\sigma(w)$ from 1 to $w$ in the Cayley graph $\Gamma_{X}\left(G_{q}\right)$ of $G_{q}$ for every $w \in F$. Define paths $\sigma(g, h)$ by taking equivariant lines; define

$$
\sigma(g, h)(t):=g \cdot \sigma\left(1, g^{-1} h\right)(t)=g \cdot \sigma\left(g^{-1} h\right)(t) \quad \forall g, h \in G_{q}
$$


Theorem 3.2. The paths $\sigma(g)$ are recursive (i.e. $|\sigma(g)| \leqq f(d(1, g)))$ with a function $f(x)=2 x^{2}+3 x$ for $q>1$ and $f(x)=x^{2}+x$ for $q=1$.

Proof. The relations in $P_{q}$ say that $z$ commutes with $x$ and $y$, in particular any power of $z$ can be shifted to any place in a given word, and that $x$ commutes with $y^{q}$ at the expense of introducing $z$ or $z^{-1}$.

For $q>1$, let $w \equiv \sigma(g) \equiv y^{s} x^{r 1} y^{s_{1}} x^{r_{2}} \ldots y^{s_{m-1}} x^{r_{m}} y^{p^{p}} z^{n} \in F$ be the normal form for $g$. We observe first that

$$
d(1, g) \geqq \sum_{i=1}^{m}\left|r_{i}\right|+\sum_{i=1}^{m-1}\left|s_{i}\right|+|s|
$$

This is due to the fact that the exponents of the $y$-powers which occur in $w$ can only be changed by adding multiples of $q$ (The relations (5) allow one to permute powers of $x$ with powers of $y^{q}$ ). However, the range for $s_{i}$ and $s$ in the normal form $w$ is such that $\left|s_{i}\right|$ and $|s|$ can not decrease under these changes. The same argument also shows that $d(1, g) \geqq \sum_{i=1}^{m}\left|r_{i}\right|+\sum_{i=1}^{m-1}\left|s_{i}\right|+|s|+\max \left\{|p|-\left(\sum\left|s_{i}\right|+|s|\right), 0\right\}$, which implies:

$$
d(1, g) \geqq|p|
$$

Therefore $\sum_{i=1}^{m}\left|r_{i}\right|+\sum_{i=1}^{m-1}\left|s_{i}\right|+|s|+|p| \leqq 2 d(1, g)$. In order to prove $|w|=\sum_{i=1}^{m}\left|r_{i}\right|+$ $\sum_{i=1}^{m-1}\left|s_{i}\right|+|s|+|p|+|n| \leqq f(d(1, g))=3 d(1, g)+2 d^{2}(1, g)$, we only need to show that $|n| \leqq d(1, g)+2 d^{2}(1, g)$ :

We claim that

$$
\begin{gathered}
d(1, g) \geqq \sum_{i=1}^{m}\left|r_{i}\right|+\sum_{i=1}^{m-1}\left|s_{i}\right|+|s| \\
+\min \left\{\max \left[|n|-\left(\sum_{i=1}^{m}\left|r_{i}\right|+|r|\right)\left[\left(\sum_{i=1}^{m-1}\left|s_{i}\right|+|s|+|p|\right)|q+| l \mid\right], 0\right]+2|r|+2 q|l|\right\}
\end{gathered}
$$

where the minimum ranges over $|r|$ and $|l|$. If $|n| \leqq\left(\sum_{i=1}^{m}\left|r_{i}\right|\right)\left(\sum_{i=1}^{m-1}\left|s_{i}\right|+|s|+|p|\right) / q$ the minimum term on the right hand will be 0 and the inequality holds by (9). If $|n|>\left(\sum_{i=1}^{m}\left|r_{\mathrm{i}}\right|\right)\left(\sum_{i=1}^{m-1}\left|s_{\mathrm{i}}\right|+|\mathrm{s}|+|\mathrm{p}|\right) / q$ we observe first that $|n|$ may decrease by at most $k|| l \mid$ if a power $y^{q l}$ is pushed across a power $x^{k}$ in $w$.

If we do not introduce new powers of $x$ or $y^{q}$ by inserting $x^{r} x^{-r}$ or $y^{q l} y^{-q l}$ into the word, the amount by which $|n|$ may be decreased by means of permuting powers of $x$ with powers of $y^{q}$ is clearly bounded by $\sum_{i=1}^{m}\left|r_{i}\right|\left(\sum_{i=1}^{m-1}\left|s_{i}\right|+|s|+|p|\right) / q$. This coarse estimate stems from the following fact: Among all words in $x$ and $y$ whose sum of absolute values of $x$-exponents and sum of absolute values of $y$-exponents is the same as for $w, y^{\sum\left|s_{i}\right|+|s|+|p|} x^{\sum\left|r_{i}\right|}$ can absorb the largest powers $z^{n^{\prime}}$ or $z^{-n^{\prime}}$ by permuting powers of $x$ with powers of $y^{q}$. 
If we prolong the word by inserting $x^{r} x^{-r}$ and $y^{q l} y^{-q l}$ at suitable places, the amount by which $|n|$ can be decreased by means of (5) is bounded by $\left(\sum_{i=1}^{m}\left|r_{i}\right|+|r|\right)\left[\left(\sum_{i=1}^{m-1}\left|s_{i}\right|+|s|+|p|\right) / q+|l|\right] ;$ and, at the same time, the length of the $x-y$-part of the word increases by $2|r|+2 q|l|$. This explains inequality (11).

Now, let $\left|r_{0}\right|$ and $\left|l_{0}\right|$ be the values for $|r|$ and $|l|$ for which the minimum occurs in (11). Then $d^{2}(1, g) \geqq\left(\sum_{i=1}^{m}\left|r_{i}\right|+2 \mid r_{0}\right)\left(\sum_{i=1}^{m-1}\left|s_{i}\right|+|s|+2\left|l_{0}\right|\right)$, and, by $(10), d^{2}(1, g) \geqq\left(\sum\left|r_{i}\right|+2\left|r_{0}\right|\right)|p|$ which implies $2 d^{2}(1, g) \geqq\left(\sum_{i=1}^{m}\left|r_{i}\right|+\left|r_{0}\right|\right)\left[\left(\sum_{i=1}^{m-1}\left|s_{i}\right|+|s|+|p|\right) / q+\left|l_{0}\right|\right]$. Therefore, by (11) again, $|n| \leqq d(1, g)+2 d^{2}(1, g)$ which proves the theorem for $q>1$.

For $q=1$ the proof is similar, but easier. Let $\sigma(g) \equiv x^{r} y^{s} z^{n}$. It is clear that $d(1, g) \geqq|r|+$ $|s|$. If $|n| \leqq|r|+|s|$, then $d(1, g)+d(1, g)^{2} \geqq|\sigma(g)|$; if $|n|>|r|+|s|$, then, by the same ideas as in the proof for $q>1, d(1, g) \geqq|r|+|s|+\min \left\{\max \left[|n|-\left(|r|+\left|r^{\prime}\right|\right)\left(|s|+\left|s^{\prime}\right|\right), 0\right]+2\left|r^{\prime}\right|+2\left|s^{\prime}\right|\right\}$ where the minimum ranges over the values of $\left|r^{\prime}\right|$ and $\left|s^{\prime}\right|$. Let $\left|r_{0}^{\prime}\right|$ and $\left|s_{0}^{\prime}\right|$ be the values for which the minimum occurs, then $|r|+|s|+|n| \geqq d(1, g)+\left(|r|+\mid r_{0}^{\prime}\right)\left(|s|+\left|s_{0}^{\prime}\right|\right) \geqq d(1, g)+$ $d^{2}(1, g)$.

Theorem 3.3. $\sigma(g, h)$ defines a bicombing of narrow shape with constants $M=24 q+18$ and $k=11 / 5$.

Proof. Recall that a recursive $\sigma$ is of narrow shape, if there exists an integer $M>1$ and a real $k>2$, such that for all $g, h \in G$ and $a, b \in X^{ \pm 1} \cup\{1\}$

$$
|\sigma(\sigma(g, h)(t), \sigma(g a, h b)(t))| \leqq \max ((|\sigma(g, h)|+|\sigma(g a, h b)|) / k, M / 2)
$$

holds for all integers $t \in[0, \infty$ [. Since the bicombing is equivariant, it suffices to show this inequality for $g=1$.

For $q>1$ let $v \in F$ be in normal form $v \equiv y^{s} x^{r_{1}} y^{s_{1}} x^{r_{2}} \ldots y^{s_{m-1}} x^{r_{m}} y^{p} z^{n}$, such that $v=h$ in $G_{q}(\sigma(1, h) \equiv v)$. Let $w$ be the group element $a^{-1} v b$ brought into normal form $(\sigma(a, v b) \equiv w)$ (see fig. 2).

Now calculate the length of the bicombing lines (the combing distance) between these two paths $w, v$ in $\Gamma_{X}\left(G_{q}\right)$. Call the maximal combing distance between two such paths $\delta(\sigma, w, v)$.

If $a=1$ and $b \in\left\{1, z^{ \pm 1}\right\}$, then $\delta(\sigma, w, v) \leqq 1$. If $a=1$ and $b \in\left\{y^{ \pm 1}\right\}$ then $\delta(\sigma, w, v)=2$.

If $a=1$ and $b \in\left\{x^{\varepsilon}\right\}(\varepsilon= \pm 1)$, then $\delta(\sigma, w, v) \leqq|l|+q+1$, where $l$ is such that $-q / 2+1 \leqq$ $p-l q \leqq q / 2$ for $q$ even and $-(q-1) / 2 \leqq p-l q \leqq(q-1) / 2$ otherwise. To see this, observe that $v$ ends with $y^{p} z^{n}$ but $w$ ends with $x^{\varepsilon} y^{q l} z^{n-e l}$. Since $|w|+|v| \geqq 2 q|l|$ we get for $q \geqq 2$ and $\delta(\sigma, w, v)>M / 2:(|w|+|v|) / k>\delta(\sigma, w, v)$.

There are a few more cases which are relatively easy. The most critical case which requires the sharpest estimates occurs if $a=y^{\varepsilon}, b=x^{\alpha}$ with $\alpha, \varepsilon \in\{ \pm 1\}$; in particular if $y^{s}$ is at the boundary of its range to which it is restricted by the normal form, and the premultiplication by $a^{-1}=y^{-\varepsilon}$ moves it out of this range, as, for example, in the case $\varepsilon=-1, s=q / 2$ and $q$ even (the other cases can be treated similarly).

In this case $v \equiv y^{q / 2} x^{r_{1}} y^{s_{1}} x^{r_{2}} \ldots y^{s_{m-1}} x^{r_{m}} y^{p^{n}} z^{n}$ and

$$
w \equiv y^{-q / 2+1} x^{r_{1}} y^{s_{1}} x^{r_{2}} \ldots y^{s_{m-1}} x^{r_{m}} y^{p-l q} x^{\alpha} y^{(l+1) q} z^{n-\sum r_{i}-\alpha(l+1)}
$$




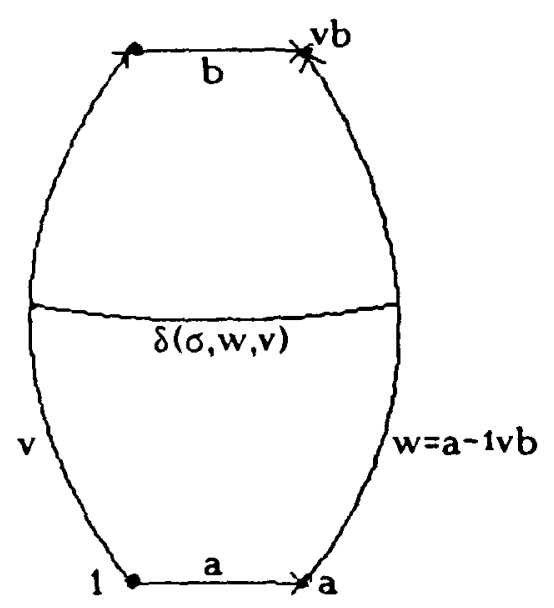

FIGURE 2. Close bicombing lines.

where $l$ is as above. Using the rule $|a|+|a-b| \geqq|b|$ we obtain the estimate: $|w|+|v| \geqq 2 q|l|$ $+2 \sum\left|r_{\mathrm{i}}\right|+\left|\sum r_{i}+\alpha(l+1)\right|$. A careful study of the lengths of the combing distances shows that

$$
\delta(\sigma, w, v) \leqq \max \left\{\max _{j \leqq m}\left|\sum_{i=1}^{j} r_{i}\right|+1,\left|\sum_{i=1}^{m} r_{i}\right|+|l|+3 q+2\right\} \leqq\left(\sum_{i=1}^{m}\left|r_{i}\right|+\left|\sum_{i=1}^{m} r_{i}\right|\right) / 2+|l|+3 q+2
$$

Since $q \geqq 2$ and $k=11 / 5,(|w|+|v|) / k \geqq 20|l| / 11+10 \sum\left|r_{i}\right| / 11+5\left|\sum r_{i}+\alpha(l+1)\right| / 11$. We will show that the right hand side is $\geqq\left(\sum_{i=1}^{m}\left|r_{i}\right|+\left|\sum_{i=1}^{m-1} r_{i}\right|\right) / 2+|l|+3 q+2$ whenever $\delta(\sigma, w, v)>M / 2$ (which, by the above estimate for $\delta(\sigma, w, v)$, proves the theorem for this case). This is equivalent to:

$$
9|l|+10 \sum\left|r_{i}\right|+5\left|\sum r_{i}+\alpha(l+1)\right| \geqq 11 \sum\left|r_{i}\right| / 2+11\left|\sum r_{i}\right| / 2+33 q+22 .
$$

The left hand side can be simplified by the following estimates: $5|l|+5\left|\sum r_{i}+\alpha+\alpha\right| \geqq$ $5\left|\sum r_{i}+\alpha\right| \geqq 5\left|\sum r_{i}\right|-5$, and $10 \sum\left|r_{i}\right|+5\left|\sum r_{i}\right| \geqq 19 \sum\left|r_{i}\right| / 2+11\left|\sum r_{i}\right| / 2 \geqq 4 \sum\left|r_{i}\right|+11\left|\sum r_{i}\right| / 2$. Therefore the above inequality follows from $4\left(|l|+\sum\left|r_{i}\right|\right) \geqq 33 q+27$, which follows from $\delta(\sigma, w, v)>M / 2$ using the value $M=24 q+18$ and the estimate $\delta(\sigma, w, v) \leqq\left(\sum\left|r_{i}\right|+\left|\sum r_{i}\right|\right) /$ $2+|l|+3 q+2 \leqq \sum\left|r_{i}\right|+3 q+2$.

The proof for $q=1$ is much simpler and left to the reader.

In the following we use Cockcroft 2-complexes to get lower bounds for isoperimetric functions. This idea is due to S. Gersten [6].

Theorem 3.4. $G_{q}$ has no quadratic isoperimetric inequality and therefore no combing in the sense of Short [10]. 
Proof. There is a van Kampen diagram for $w_{n} \equiv\left[x^{n}, y^{q n}\right] \cdot\left[y^{-q n}, x^{-n}\right]$ in $G_{q}$, which has $n^{3}$ more 2-cells $[x, z]$ of positive then of negative type. W. A. Bogley proves in [3], that the corresponding 2-complex is Cockcroft. So each $\pi_{2}$-element has the same number of positive as of negative 2-cells $[x, z]$, which proves that every van Kampen diagram for $w_{n}$ will contain at least $n^{3} 2$-cells $[x, z]$ and so proves the theorem.

\section{REFERENCES}

1. J. M. Alonso, Combings of groups, in: Algorithms and Classification in Combinatorial Group Theory, G. Baumslag and C. F. Miller III, eds. (Springer Verlag, MSRI Publ., 1991).

2. J. M. Alonso and M. R. Bridson, Semihyperbolic groups (preprint, Cornell University, 1990).

3. W. Bogley, Unions of Cockcroft two-complexes, preprint, 1991.

4. M. BRIDSON, On the geometry of normal forms in discrete groups (preprint, Princeton University, 1992).

5. David B. H. Epstein, J. W. Cannon, D. F. Holt, S. V. F. Levy, M. S. Paterson and W. P. ThURSTON, Word processing in groups (Jones and Bartlett, 1992).

6. S. Gersten, Dehn functions and $l_{1}$-norms of finite presentations, in: Proceedings of the Workshop on Algorithmic Problems, C. F. Miller III and G. Baumslag, eds. (Springer Verlag, 1991).

7. S. Gersten, Isoperimetric and isodiametric functions of finite presentations (preprint, University of Utah, 1991).

8. M. Gromov, Hyperbolic groups, in: Essays in group theory (Springer Verlag, 1987), 75-263.

9. R. Lyndon and P. Schupp, Combinatorial Group Theory (Springer Verlag, Berlin, 1977). (1990).

10. H. Sногт, Groups and combings, Laboratoire de Math., Ecole Normale Sup. de Lyon

Institut F. Didaktik der Mathematik

J.-W.-GOETHE UNIVERSITÄT

SenCKenberganlage 9

6000 FrankFURT/M.

West Germany
Department of Mathematics

Northern ARIzONA UnIVERSITY

FlagstafF AZ 86011

USA 